Pacific

Journal of

Mathematics

THE COBORDISM OF ORIENTED THREE DIMENSIONAL ORBIFOLDS

K.S. DRUSChEL 


\title{
THE COBORDISM OF ORIENTED THREE DIMENSIONAL ORBIFOLDS
}

\author{
K.S. DRUSCHEL
}

\begin{abstract}
In this paper we construct a decomposition for oriented orbifold cobordism and apply it to establish that every oriented three dimensional orbifold bounds an oriented four orbifold.
\end{abstract}

\section{Introduction.}

In a previous paper [2], using orbifold Pontrjagin numbers, we had established that rationally every odd dimensional oriented orbifold bounds and, also, if an orbifold bounds, then some multiple of it bounds with no increase in the set of local groups. The proof involves systematically making the orbifold rationally cobordant to one with a smaller set of local groups. Thus to study torsion in orbifold cobordism one must gain control of the way one introduces new local groups when one tries to make the singularities associated with the original orbifold bound. In Section one we collect some definitions associated with orbifolds and their cobordism. In Section two we consider various orbifold cobordism groups with restrictions on the set of local groups and fit these into a commutative diagram with cobordism groups of certain orbibundles over manifolds. This is much in the spirit of [1]. In Section three we apply this to show that every two dimensional and every three dimensional orbifold bounds.

\section{Orbifold Cobordism.}

In this section we briefly review some material associated with orbifolds and cobordism.

An orbifold chart on a topological space $X$ is defined by a triple $(U, G, \pi)$ where $U$ is an open subset of $\mathbf{R}^{n}, G$ is a finite group acting effectively and differentiably on $U$, and $\pi: U \rightarrow X$ factors as $h p_{U}$ where $p_{U}$ is the natural orbit map and $h$ is open and injective. Two charts $\left(U_{i}, G_{i}, \pi_{i}\right), i=1,2$, are compatible if for $x \in \pi_{1}\left(U_{1}\right) \cap \pi_{2}\left(U_{2}\right)$ and $\bar{x}$ a lift of $x$ in $U_{1}$ there is an open set $U \subset U_{1}$ about $\bar{x}$ and a diffeomorphism $f: U \rightarrow U^{\prime}$ onto an open subset $U^{\prime} \subset U_{2}$ such that $\pi_{2} f=\pi_{1}$. Such a map is called an overlap map. An $\mathrm{n}$ dimensional orbifold $Q$ is defined by a Hausdorff underlying space, denoted $|Q|$, together with a maximal atlas of compatible orbifold charts on $|Q|$. 
An $n$ dimensional orbifold with boundary is defined as above except that $U$ is an open subset of $\mathbf{H}^{n}=\left\{\left(x_{1}, x_{2}, \ldots, x_{n}\right) \in \mathbf{R}^{n}: x_{n} \geq 0\right\}$. If $W$ is an orbifold with boundary, the orbifold boundary of $W$, denoted $\delta_{o} W$, has underlying space $\left\{w \in|W|: w=\pi_{\alpha}(x), x \in \delta \mathbf{H}^{n}\right\}$ where $\pi_{\alpha}$ is the map for some chart for $W$. An orbifold $Q$ is orientable if it has an atlas $\left\{\left(U_{\alpha}, G_{\alpha}, \pi_{\alpha}\right), \alpha \in \Lambda\right\}$ such that the $U_{\alpha}$ 's can all be oriented consistently with the $G_{\alpha}^{\prime} s$ and the overlap maps orientation preserving. It is oriented once one chooses a consistent orientation of the charts.

Two compact, oriented $n$ dimensional orbifolds $Q_{1}, Q_{2}$, are orbifold cobordant if there is an oriented orbifold with boundary $W$, such that $\delta_{o} W=$ $Q_{1} \amalg-Q_{2}$ with the appropriate orientations. As with manifold cobordism $\left(\Omega_{n}\right)$ one has the group of cobordism classes of oriented $n$ dimensional orbifolds, which we denote by $\Omega_{n, \text { orb }}$.

For $x \in|Q|$ the local group at $x$ is the isotropy group $G_{\hat{x}}$ for a chart $(U, G, \pi)$ with $\hat{x} \in U$ a lift of $x$. Using the differential, $G_{x}$ may be considered as a subgroup of $G l(n, \mathbf{R})$ and is then well defined up to conjugacy. We denote this conjugacy class by $\left(G_{x}\right)$ and the set of conjugacy classes of local groups for $Q$ by $\mathcal{G}_{Q}$. If $Q$ has a Riemannian metric, which is possible if $|Q|$ is compact, then we can view $G_{x}$ as a subgroup of $O(n)$, and then, if $Q$ is oriented, as a subgroup of $S O(n)$. For a subgroup $K$ of $G l(n, \mathbf{R})$ let $j$ be the dimension of the subspace of $\mathbf{R}^{n}$ on which $K$ acts trivially. The degree of $K(\operatorname{deg} K)$ is $n-j$. For $(H) \in \mathcal{G}_{Q}$ the $H$ singular set of $Q$, denoted $Q^{H}$, is defined to be the set $\left\{x \in Q \mid(H)<\left(G_{x}\right)\right\}$.

We will be particularly interested in two examples of $(F, G)$ orbibundles $[2,1.8]$. For an orbifold $Q$ with atlas $\left\{\left(U_{\alpha}, G_{\alpha}, \pi_{\alpha}\right), \alpha \in \Lambda\right\}$ an $l$ dimensional orbivector bundle $\Psi$ over $Q$ has total space (Tot $\Psi$ ) an orbifold $E$ which locally looks like $U_{\alpha} \times_{G_{\alpha}} \mathbf{R}^{l}$ where the action of $G_{\alpha}$ and the overlap maps for $E$ are linear on the second factor. If these are also orthogonal, then we can associate with $\Psi$ the sphere and disc bundles $S \Psi$ and $D \Psi$. If they are orientation preserving then we say $\Psi$ is oriented.

To study the $H$ singular set in an orbifold $Q$ we look at the normal bundle $\nu_{H}\left[\mathbf{2}\right.$, p. 310], an $\left(\mathbf{R}^{l} / H, N_{G l(l, \mathbf{R})}(H) / H\right)$ bundle over an orbifold $Q_{H}$. Here $N_{K}(G)$ denotes the normalizer of the group $G$ in $K, l$ is the degree of $H$, and $Q_{H}$ is the best orbifold approximation to $Q^{H}[\mathbf{2}, 2.9]$. When $H$ is maximal in $\mathcal{G}_{Q} Q^{H}=Q_{H}$ and Tot $\nu_{H}$ is diffeomorphic to a neighborhood of $Q^{H}$ in $Q$. When $|Q|$ is compact we can replace $G l(l, \mathbf{R})$ with $O(l)$ and have associated sphere and disc bundles, $S \nu_{H}$ and $D \nu_{H}$.

\section{A Decomposition for Oriented Orbifold Cobordism.}

Throughout this we assume that every orbifold is compact, smooth, and oriented unless stated otherwise.

From $[\mathbf{2}, 2.14]$ and $[\mathbf{2}, 1.15]$ we conclude the following. 
Lemma 2.1. If an orbifold $Q$ bounds then some multiple $s$ of $Q$ bounds an orbifold $W$ such that $\mathcal{G}_{Q}=\mathcal{G}_{W}$, i.e. $s Q$ bounds with no increase in local groups.

Now suppose $(H)$ is maximal in $\mathcal{G}_{Q}$. Then $Q^{H}$ is a manifold and the normal bundle to $Q^{H}$ in $Q$ is an ordinary $\left(R^{n} / H, N_{O(n)}(H) / H\right)$ bundle. [2, p. 305]. (Here $n$ is the degree of $H$.) Also when $s Q=\delta_{\text {orb }} W$ with $\mathcal{G}_{Q}=\mathcal{G}_{W}$, we have the normal bundle to $W^{H}$ in $W$ is an ordinary $\left(R^{n} / H, N_{O(n)}(H) / H\right)$ bundle over the manifold $W^{H}$ and an extension of the normal bundle to $s Q$. Hence one wants to first consider the following.

Definition 2.2. Suppose $H<S O(n)$ has degree $n$. We let $B_{k}(H)$ be the set of cobordism classes of $\left(R^{n} / H, N_{O(n)}(H) / H\right)$ bundles over $k$-dimensional manifolds where we require that the total space of the bundle is oriented.

By using the classifying maps for such bundles we have that $B_{k}(H)$ is isomorphic to $\Omega_{k, t}\left(B N_{O(n)}(H) / H\right)[2,2.3]\left(\right.$ or $\Omega_{k}\left(B N_{O(n)}(H) / H\right)$ if $N_{O(n)}(H)$ $<S O(n))$. Here $\Omega_{k}(X)$ denotes the group of bordism classes of maps from oriented $k$ dimensional manifolds into the space $X$. If there is some canonical orbibundle $\Gamma$ over $X$ then $\Omega_{k, t}(X)$ consists of bordism classes of maps $f: M^{k} \rightarrow X$ such that $f^{*} \Gamma \oplus T M$ is oriented. $\Omega_{k, t}\left(B N_{O(n)}(H) / H\right)$ is rationally isomorphic to the twisted homology $H_{*, t}\left(B N_{O(n)}(H) / H, \Omega_{*}\right)$. [2, $2.4]$.

Once we are asking whether an orbifold $Q$ bounds exactly and not just rationally, we find that we can no longer restrict the local groups of the bounded orbifold $W$ to be those of $Q$. In other words if $Q=\delta_{\text {orb }} W$ it may be necessary that $\mathcal{G}_{W}$ is strictly larger than $\mathcal{G}_{Q}$. This is born out by the following illustration. This illustration is due to Quach Ngoc Du, as communicated to me by Mike Davis, as is a generalization to prove that an oriented two dimensional orbifold bounds.

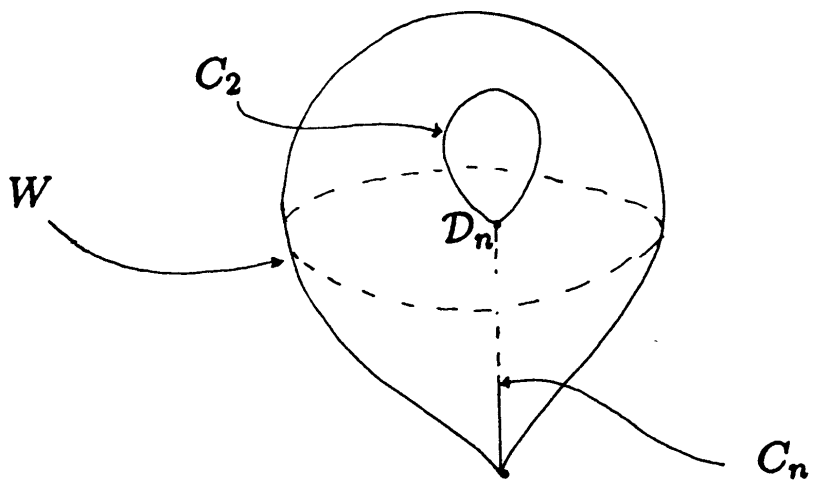

Figure 2.1. 
$W$ is topologically a three disc. As an orbifold it has singular set a half open line segment labelled $C_{n}$, the cyclic group of order $n$, a point labelled by the dihedral group $\mathcal{D}_{n}$, and an open line segment labelled $C_{2}$. The orbifold boundary of $W$ is a teardrop orbifold with singular set a point labelled $C_{n}$. For $H=C_{n}, W^{H}$ is a folded closed line segment with its fold point labelled $\mathrm{C}_{2}$.

Thus we see that $W^{H}$ need not be a manifold and that $B_{k}(H)$ is too restrictive. However, in general, if $Q^{H}$ bounds an orbifold $T$ and the $\left(\mathbf{R}^{n} / H\right.$, $\left.N_{O(n)}(H) / H\right)$ bundle over $Q^{H}$ extends to an $\left(\mathbf{R}^{n} / H, N_{O(n)}(H) / H\right)$ orbibundle $\Psi$ over $T$, then $Q$ is orbifold cobordant to $Q-\left(\operatorname{int}\left(D \nu_{H}\right)\right) \cup_{S \nu_{H}} S \Psi$. (See the proof of 2.8.) For the resulting orbifold one has local groups those of $Q$ with $H$ thrown out, and then those of $S \Psi$ added in. One wants to gain some control of which local groups are added in by $S \Psi$ so we consider the following.

Let $\mathcal{F}$ denote a set of conjugacy classes of finite subgroups of $S O(m) . \mathcal{F}$ is closed if whenever $(K) \in \mathcal{F}$ then $(L) \in \mathcal{F}$ for every $(L) \in \mathcal{G}_{R^{m}} / K$. Let $H$ be a finite linear group of degree $l$ and $\hat{\mathcal{F}} \supset \mathcal{F}$ indicate any two closed sets of classes of finite subgroups of $S O(l+k+1)$ and $S O(l+k)$, respectively, with $(H) \in \mathcal{F}$.

Definition 2.3. Suppose $(H)$ is maximal in $\mathcal{F}$. Define $B_{k}(H, \mathcal{F}, \hat{\mathcal{F}})$ to be the equivalence classes of pairs $(M, \nu)$ where $\nu$ is an $\left(\mathbf{R}^{l} / H, N_{O(l)}(H) / H\right)$ bundle over a k dimensional manifold $M$ with the total space of $\nu$ oriented. The equivalence relation is defined by $\left(M_{1}, \nu_{1}\right) \sim\left(M_{2}, \nu_{2}\right)$ if there is an $\left(\mathbf{R}^{l} / H, N_{O(l)}(H) / H\right)$ orbibundle $\Psi$ over an orbifold $W$ so that

i) $\operatorname{Tot} \Psi$ is oriented

ii) $\mathcal{G}_{T o t} \Psi \subset \hat{\mathcal{F}}, \mathcal{G}_{S \Psi} \subset \mathcal{F}-(H)$

iii) $M_{1} \amalg M_{2}=\delta_{\text {orb }} W$ (not necessarily orientably)

iv) $\Psi_{\mid M_{i}}=\nu_{i}$ and Tot $\nu_{i}$ has the inherited orientation.

Note that if $G \in \mathcal{G}_{\text {Tot } \Gamma}$ for $\Gamma$ an $\left(\mathbf{R}^{l} / H, N_{O(l)}(H) / H\right)$ orbibundle over an orbifold $Q^{n}$ with $T o t \Gamma$ oriented then $G<N_{S O(n+l)}(H)$ and hence $G<N_{O(l)}(H) \oplus O(n)$. Also, tracing through the definition of an $\left(\mathbf{R}^{l} / H\right.$, $\left.N_{O(l)}(H) / H\right)$ orbibundle one sees that ker $\rho_{2 \mid G}=H$. Here $\rho_{i}: O(l) \oplus$ $O(n) \rightarrow O(s), i=1,2$ is projection onto the first, respectively second factor with $s=l$, respectively $n$.

For $\Psi$ as in the above definition, the second part of ii) implies that if $G \in \mathcal{G}_{T o t} \Psi$ and $(K) \in \mathcal{G}_{\mathbf{R}^{l+k+1} / G}$ with $\operatorname{deg} \rho_{1}(K)<l$ (so $K$ shows up as a local group for $S \Psi)$ then $(K) \in \mathcal{F}-(H)$. Thus we have the following definition.

Definition 2.4. Let $\mathcal{F}$ be as above and $H<G<N_{O(l)}(H) \oplus O(k+1)$ with ker $\rho_{2 \mid G}=H$. Then $G$ satisfies condition $S_{\mathcal{F}, H}$ if for every $(K) \in \mathcal{G}_{\mathbf{R}^{l+k+1} / G}$ with $\operatorname{deg} \rho_{1}(K)<l,(K) \in \mathcal{F}-(H)$. 
Now let $\mathcal{F} \subset \hat{\mathcal{F}}$ be a pair of closed sets of conjugacy classes of finite subgroups of $S O(n)$, respectively $S O(n+1)$.

Definition 2.5. Let $\Omega_{n \text {,orb }}(\mathcal{F}, \hat{\mathcal{F}})=\left\{Q^{n}: \mathcal{G}_{Q} \subset \mathcal{F}\right\} / \sim$, where $Q_{1} \sim Q_{2}$ if there is an orbifold $W$ with $\mathcal{G}_{W} \subset \hat{\mathcal{F}}$ and $Q_{1} \amalg-Q_{2}$ the oriented orbifold boundary of $W$.

Let $\mathcal{F}_{\text {all }, m}$ be the set of all conjugacy classes of finite subgroups of $S O(m)$ and $\Omega_{n, \text { orb }}(\mathcal{F})=\Omega_{n, \text { orb }}\left(\mathcal{F}, \mathcal{F}_{\text {all }, n+1}\right)$.

\section{Proposition 2.6.}

i) $\Omega_{n, \text { orb }}=\Omega_{n, \text { orb }}\left(\mathcal{F}_{\text {all }, n}\right)$.

ii) Since $\mathcal{G}_{Q}$ is finite for a compact orbifold $Q, \Omega_{n, \text { orb }}(\mathcal{F}, \hat{\mathcal{F}})=\cup \Omega_{n, \text { orb }}\left(\mathcal{F}_{\alpha}\right.$, $\hat{\mathcal{F}})$ where the union is over all closed finite subsets $\mathcal{F}_{\alpha}$ of $\mathcal{F}$.

iii) If $\mathcal{F}_{1} \subset \mathcal{F}_{2} \subset \hat{\mathcal{F}}$ then $\Omega_{n, \text { orb }}\left(\mathcal{F}_{1}, \hat{\mathcal{F}}\right) \subset \Omega_{n, \text { orb }}\left(\mathcal{F}_{2}, \hat{\mathcal{F}}\right)$.

iv) If $\mathcal{F} \subset \hat{\mathcal{F}}_{1} \subset \hat{\mathcal{F}}_{2}$ then the natural map $\tau: \Omega_{n, \mathrm{orb}}\left(\mathcal{F}, \hat{\mathcal{F}}_{1}\right) \rightarrow \Omega_{n, \mathrm{orb}}\left(\mathcal{F}, \hat{\mathcal{F}}_{2}\right)$ is onto.

From Lemma 2.1 we conclude:

Proposition 2.7. ker $\tau \subset$ torsion $\left(\Omega_{n, \text { orb }}\left(\mathcal{F}, \hat{\mathcal{F}}_{1}\right)\right)$.

The various cobordism groups, $B_{k}(H, \mathcal{F}, \hat{\mathcal{F}})$, of $R^{n} / H$ bundles, and the orbifold cobordism groups with various restrictions on the local groups fit together according to the following commutative diagram.

Here $g_{1}, g_{2}, g_{3}$ are inclusion maps, $h_{i}[Q]=\left[\left(Q^{H}, \nu_{H}\right)\right]$ and $k_{i}[(M, \nu)]=$ $[S \nu]$, and $[(M, \nu)]$ or $[Q]$ denotes the appropriate cobordism class.

Also we must assume that $H$ is maximal in $\mathcal{F}$ and if $L \in \hat{\mathcal{F}}$ with $H<L$ then $N_{L}(H)$ has condition $S_{\mathcal{F}, H}$ as in Definition 2.4. The latter ensures that $h_{2}$ is well defined. Indeed, suppose $\left[Q_{i}\right] \in \Omega_{n \text {,orb }}(\mathcal{F}, \hat{\mathcal{F}}), i=1$, 2 , with $\left[Q_{1}\right]=\left[Q_{2}\right]$ via the orbifold with boundary $W$. If the normal bundle of $W_{H}$ in $W$ is denoted by $\Psi$, then the local groups of Tot $\Psi$ are of the form $N_{G_{x}}(H), x \in W_{H}$, and condition $S_{\mathcal{F}, H}$ guarantees that $\left[\left(Q_{1}^{H}, \nu_{H}\left(Q_{1}^{H}\right)\right)\right]=$ $\left[\left(Q_{2}^{H}, \nu_{H}\left(Q_{2}^{H}\right)\right)\right]$ in $B_{n-\operatorname{deg} H}(H, \mathcal{F}, \hat{\mathcal{F}})$ via $\left(W_{H}, \Psi\right)$. 


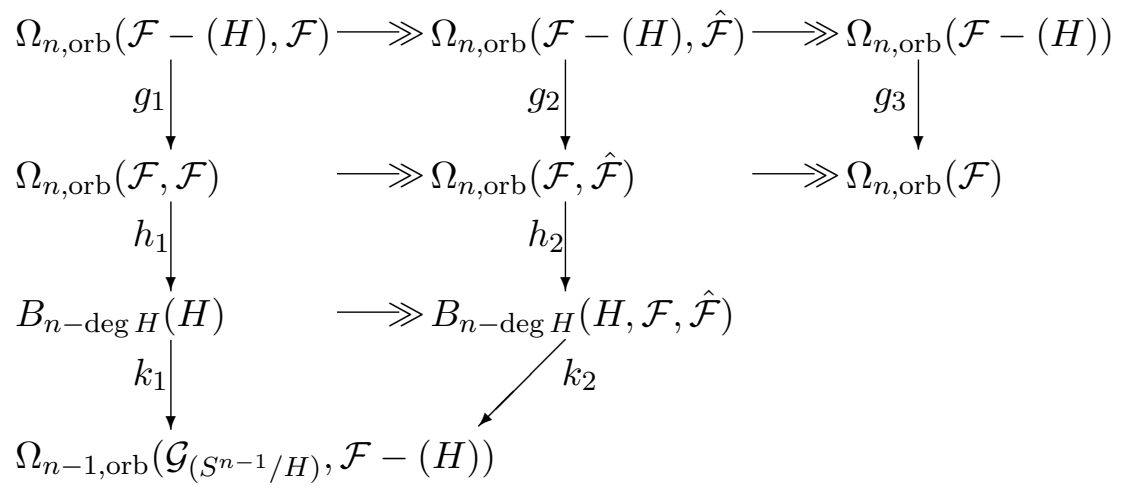

The following lemma allows us to tell when an orbifold $Q$ with local groups in $\mathcal{F}$ is cobordant to one with local groups in $\mathcal{F}-(H)$ by examining the normal bundle to $Q^{H}$. The commutivity of the diagram allows more control of the local groups in the cobordism.

Lemma 2.8. The two columns in the above diagram are exact.

Proof. To prove ker $h_{i} \subset i m g_{i}$, note that if $\left[\left(Q_{H}, \nu_{H}\right)\right]=0$ in $B_{n-\operatorname{deg} H}(H$, $\mathcal{F}, \hat{\mathcal{F}})$ via $(W, \Psi)$, then the local groups of $S \Psi$ are in $\mathcal{F}-(H)$. Thus $Q$ is cobordant to $\left(Q-D \nu_{H}\right) \cup_{S \nu_{H}} S \Psi$, whose local groups are in $\mathcal{F}-(H)$, since those of $Q-D \nu_{H}$ and $S \Psi$ are. This cobordism is via $(Q \times I) \cup_{\left(D \nu_{H}, 1\right)}$ $D \Psi$, which has local groups in $\hat{\mathcal{F}}$, since both $Q$ and $\operatorname{Tot} \Psi$ do. The reverse inclusion of image and kernal is true vacuously. We have im $h_{i} \subset$ ker $k_{i}$ since $S \nu_{H}$ bounds $Q-D \nu_{H}$. If for $[(M, \Psi)] \in B_{n-\operatorname{deg} H}(H, \mathcal{F}, \hat{\mathcal{F}}), k_{i}[(M, \Psi)]=0$, then $S \Psi=\delta_{\text {orb }} W$ with $\mathcal{G}_{W} \subset \mathcal{F}-(H)$. Hence $(M, \Psi) \sim\left(Q_{H}, \nu_{H}\right)$ where $Q=W \cup_{S \Psi} D \Psi$.

\section{Applications.}

We illustrate Lemma 2.8 with a proof of the following. Consideration of Figure 2.1 leads to an alternative proof of this proposition.

Proposition 3.1. $\Omega_{2, \text { orb }}=0$.

Proof. If $\mathcal{F}$ is a finite set of conjugacy classes of finite subgroups of $S O(2)$ it consists of cyclic subgroups, say $C_{n_{1}}, \ldots C_{n_{k}}$. Suppose $C_{2} \in \mathcal{F}$. If $H=C_{n_{i}}$, $n_{i} \neq 2$, and $\hat{\mathcal{F}}=\mathcal{F} \cup \mathcal{D}_{n_{i}}$ then $\mathcal{D}_{n_{i}}$ satisfies condition $S_{\mathcal{F}, H}$. We note 
that in general $B_{0}(H, \mathcal{F}, \hat{\mathcal{F}})$ is generated by the trivial $R^{l} / H$ bundle over a point. Here this bundle bounds the bundle $\Psi$ with total space $I \times Z_{2}$ $\left(\mathbf{R}^{2} / C_{n_{i}}\right)$ where $Z_{2}$ folds $I$ and is reflection across the $x$ axis for $\mathbf{R}^{2}$ and $\mathcal{G}_{S \Psi}=\left\{\left(C_{2}\right),(e)\right\}$. Thus $B_{0}(H, \mathcal{F}, \hat{\mathcal{F}})=0$ and $g_{i}, i=2,3$, are isomorphisms giving that $\Omega_{2, \text { orb }}(\mathcal{F}) \cong \Omega_{2, \text { orb }}\left(\mathcal{F}-\left(C_{n_{i}}\right)\right.$ ). Repeating this argument for $\mathcal{F}-\left(C_{n_{i}}\right)$ we get $\Omega_{2, \text { orb }}(\mathcal{F}) \cong \Omega_{2, \text { orb }}\left(\left(C_{2}\right),(e)\right)$. From Proposition 2.6 ii $)$ we have $\Omega_{2, \text { orb }} \cong \Omega_{2 \text {,orb }}\left(\left(C_{2}\right),(e)\right)$. Every two dimensional orbifold $Q$ with points $x_{1}, \ldots, x_{k}$ labelled $C_{2}$ is cobordant to another orbifold $Q_{1}$ with an even number of points labelled $C_{2}$ via $\left(Q \times I \coprod k\left(I \times Z_{2}\left(\mathbf{R}^{2} / C_{n_{i}}\right)\right)\right) / \sim$ where each $\left[\left(0, \mathbf{R}^{2} / C_{n_{i}}\right)\right]$ is glued to a chart neighborhood of an $x_{i}$ in $Q \times 1$. $Q_{1}$ is cobordant to a manifold with $k$ more handles than $Q$ via $Q_{1} \times I \coprod k\left(I \times Z_{2}\right.$ $\left.\left(\mathbf{R}^{2} / C_{n_{i}}\right)\right) / \sim$ where the ends of the $I \times\left(\mathbf{R}^{2} / C_{n_{i}}\right)$ 's are glued to chart neighborhoods of the singular points in $Q_{1} \times 1$. Thus $\Omega_{2, \text { orb }}\left(\left(C_{2}\right),(e)\right) \cong$ $\Omega_{2}=0$.

Theorem 3.2. Every compact, oriented, three dimensional orbifold bounds an oriented, compact orbifold.

Proof. The set of conjugacy classes in $O(3)$ of all finite subgroups of $S O(3)$ consists of two infinite families coming from the cyclic and dihedral groups, and three other classes from the tetrahedral group, $\mathcal{T}$, the octahedral group, $\mathcal{O}$, and the icosahedral group, $\mathcal{I}$.

Let $\mathcal{F}$ be any set of conjugacy classes that contains $\mathcal{I}$ and the dihedral groups $\mathcal{D}_{s}, s=2,3,5$, and $\hat{\mathcal{F}}=\mathcal{F} \cup(\overline{\mathcal{I}})$ where $\overline{\mathcal{I}}=\left\langle\mathcal{I},-\mathrm{id}_{4}\right\rangle$. Here $-\mathrm{id}_{4}$ refers to the $4 \times 4$ diagonal matrix with -1 's down the diagonal. Then $(\mathcal{F}, \hat{\mathcal{F}})$ satisfies the conditions for Lemma 2.8 with $H=\mathcal{I}$. We see that $B_{0}(\mathcal{I}, \mathcal{F}, \hat{\mathcal{F}})=0$ as the $R^{3} / \mathcal{I}$ bundle over a point bounds the $R^{3} / \mathcal{I}$ bundle $\Psi$ over $[-1,1] / Z_{2}$ where $T$ ot $\Psi=[-1,1] \times\left\langle-\operatorname{id}_{4}\right\rangle R^{3} / \mathcal{I}$ and $S \Psi$ has maximal local groups $\mathcal{D}_{s}, s=2,3,5$, and thus local groups in $\mathcal{F}-(H)$. (See Figure 3.1 below for a picture of the singular set of $S \Psi$, where the singular set of $S^{3} / \mathcal{I}$ on the left is folded by $-\mathrm{id}_{4}$ to form that of $S \Psi$.) From Lemma 2.8 we thus have $g_{2}$ is an isomorphism and then so is $g_{3}: \Omega_{3 \text {,orb }}(\mathcal{F}-(\mathcal{I})) \rightarrow \Omega_{3 \text {,orb }}(\mathcal{F})$. In particular $\Omega_{3, \text { orb }} \cong \Omega_{3, \text { orb }}\left(\mathcal{F}_{\text {all }, 3}-(\mathcal{I})\right)$.

Replacing $\mathcal{I}$ with $\mathcal{O}$ in the above paragraph and letting $s=2,3,4$ and $\mathcal{F}=\mathcal{F}_{\text {all }, 3}-(\mathcal{I})$, yields $\Omega_{3, \text { orb }}\left(\mathcal{F}_{\text {all }, 3}-(\mathcal{I})\right) \cong \Omega_{3, \text { orb }}\left(\mathcal{F}_{\text {all }, 3}-(\mathcal{I})-(\mathcal{O})\right)$. Applying this method again with $H=\mathcal{T}$ and $s=2,3$, we obtain $\Omega_{3 \text {,orb }} \cong$ $\Omega_{3, \text { orb }}\left(\mathcal{F}_{1}\right)$ where $\mathcal{F}_{1}$ consists of the classes of dihedral and cyclic groups.

We cannot use the above method to get rid of $\mathcal{D}_{n}$. Also when $H=\mathcal{D}_{n}$ and $(H) \in \mathcal{F} \subset \mathcal{F}_{1}$ the map $k_{1}: B_{0}(H) \rightarrow \Omega_{2 \text {,orb }}\left(\mathcal{G}_{S^{2} / H}, \mathcal{F}-(H)\right)$ is nonzero as $S^{2} / D_{n}$ cannot bound with local groups in $\mathcal{F}-(H)$. (Indeed the point labelled $C_{n}$ in $S^{2} / D_{n}$ can only bound a folded line segment labelled $C_{n}$ and such a line segment can only possibly fit into $\mathcal{D}_{n}, \mathcal{I}, \mathcal{O}$, or $\mathcal{T}$, none of which are in $\mathcal{F}-(H)$.) Hence, by Lemma $2.8, h_{1}: \Omega_{3, \text { orb }}(\mathcal{F}, \mathcal{F}) \rightarrow B_{0}(H)$ is the zero map. In other words, for $Q$ with local groups in $\mathcal{F}$ the normal bundle 
to $Q^{H}$ bounds in $B_{0}(H)$, meaning there are even number of points labelled $(H)$. Thus $g_{i}, i=1,2,3$, are isomorphisms. Then using Proposition 2.6 ii) we have $\Omega_{3, \text { orb }}\left(\mathcal{F}_{1}\right) \cong \Omega_{3 \text {,orb }}\left(\mathcal{F}_{2}\right)$ where $\mathcal{F}_{2}$ consists of the cyclic groups in $S O(3)$.

Now $B_{1}\left(C_{k}\right)$ is generated by $R^{2} / C_{k}$ bundles over $S^{1}$ whose total space is oriented. These bundles are trivial (since $\pi_{1}\left(B\left(N_{S O(2)}\left(C_{k}\right) / C_{k}\right)\right)=\pi_{1}\left(B S^{1}\right)$ $=0)$ and so bound $D^{2} \times \mathbf{R}^{2} / C_{k}$. This gives us that $B_{1}\left(C_{k}\right)=0$ and hence $g_{1}$ and $g_{3}$ are isomorphisms. Thus $\Omega_{3, \text { orb }} \cong \Omega_{3 \text {,orb }}\left(\mathcal{F}_{2}\right) \cong \Omega_{3}$, which is zero $[3]$.

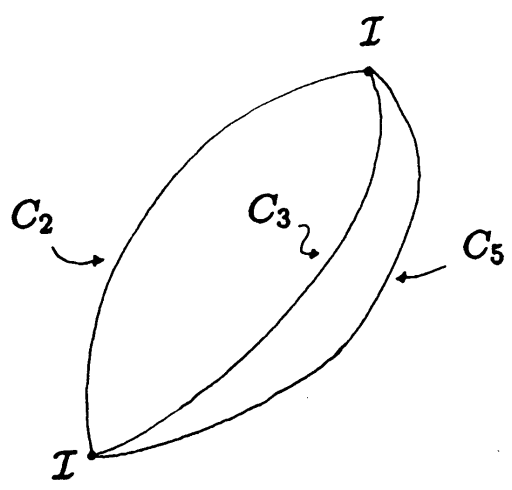

Singular set for $S^{3} / \mathcal{I}$.

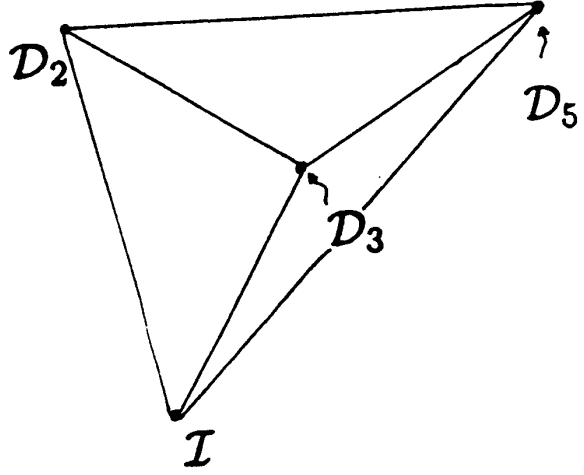

Singular set for $S^{3} /\left\langle\mathcal{I},-\mathrm{id}_{4}\right\rangle$.

Figure 3.1.

We now illustrate the proof of Theorem 3.2 by showing how a three orbifold $Q$ with singular set as in Figure 3.2 is cobordant to a three dimensional manifold. Here unlabelled arcs have singular set $C_{2}$. Figure 3.3 gives the singular set of a four dimensional orbifold with boundary which makes $Q$ cobordant to an orbifold $Q_{1}$ with no local groups $\mathcal{I}$, as in the first paragraph of the proof. In Figure 3.3 the singular set around the point labelled $\left\langle\mathcal{I},-\mathrm{id}_{4}\right\rangle$ consists of four planes labelled by $C_{k}, k=3,5,2,2$, meeting in lines labelled by $\mathcal{D}_{k}, k=3,5,2$, and $\mathcal{I}$. Figure 3.4 shows the singular set of $Q_{1}$ and another orbifold $Q_{2}$ which is cobordant to $Q_{1}$ and has dihedral and cyclic local groups. Note that there is an even number of points in $Q_{2}$ labelled by each dihedral group so, as in the third paragraph of the proof, we can systematically make $Q_{2}$ cobordant to an orbifold with fewer dihedral local groups. Figure 3.5 shows the singular sets of $Q_{3}$ and $Q_{4}$, both cobordant to $Q_{2}$. In $Q_{3}$ the dihedral groups $\mathcal{D}_{3}$ have been removed, and in $Q_{4}$ the only local dihedral groups are $\mathcal{D}_{2} . Q_{4}$ is cobordant to an orbifold $Q_{5}$ with singular set two circles labelled $C_{2}$, two labelled $C_{3}$, and one labelled $C_{5}$. This is via $\left(Q_{4} \times I\right) \amalg\left(\left(\mathbf{D}^{2} / \mathcal{D}_{2}\right) \times I\right) / \sim$ where, since $D \nu_{\mathcal{D}_{2}}\left(Q_{4}\right) \cong \mathbf{D}^{2} / \mathcal{D}_{2} \coprod \mathbf{D}^{2} / \mathcal{D}_{2}$, we 
identify $D \nu_{\mathcal{D}_{2}}\left(Q_{4}\right)$ with the ends of $\left.\left(\mathbf{D}^{2} / \mathcal{D}_{2}\right) \times I\right)$. One uses similar orbifolds with boundary for the cobordisms between $Q_{2}$ and $Q_{3}$, and then between $Q_{3}$ and $Q_{4}$. Finally, one makes $Q_{5}$ cobordant to a three manifold via the orbifold $\left(Q_{5} \times I\right) \cup_{Y} X$ where $X$ consists of a disjoint union of $\mathbf{D}^{2} \times\left(\mathbf{D}^{2} / C_{k}\right)$ 's, $k=3,3,2,2,5$, and $Y$ is diffeomorphic to a disjoint union of $S^{1} \times\left(\mathbf{D}^{2} / C_{k}\right)$ 's, $k=3,3,2,2,5$.

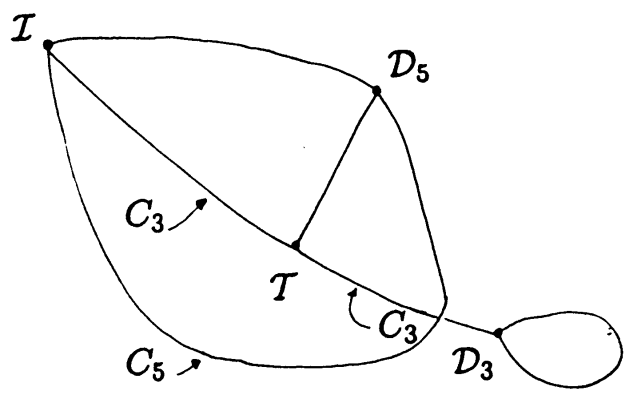

Figure 3.2. Singular set for $Q$.

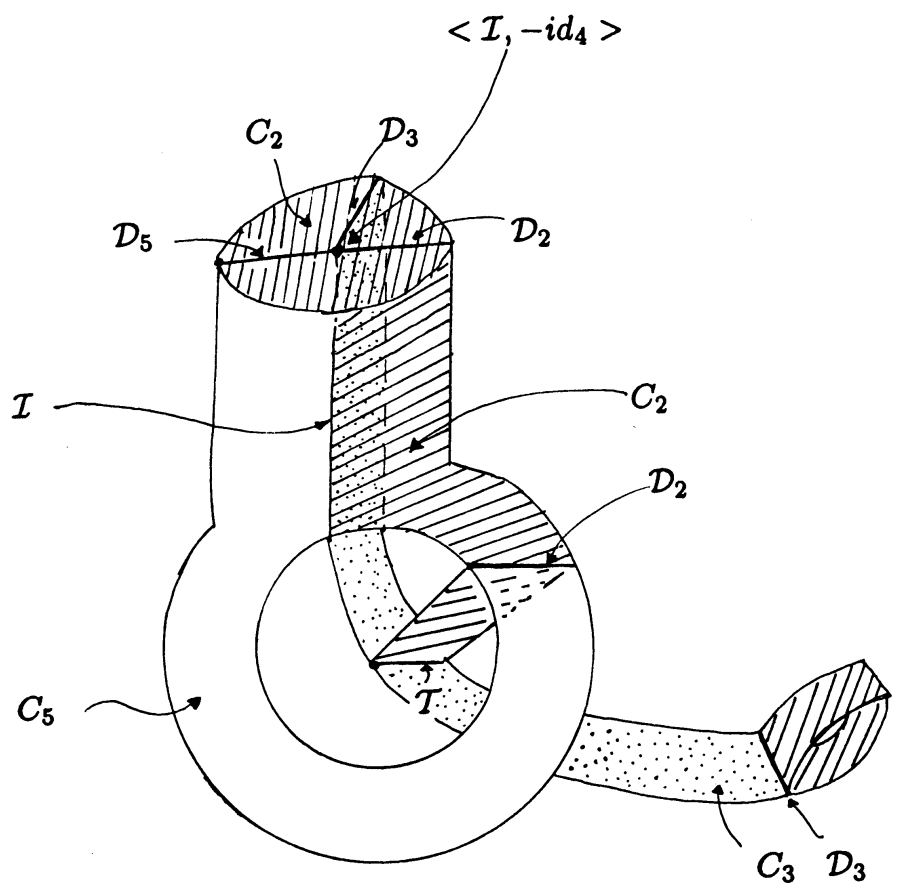

Figure 3.3. Singular set for $(Q \times I) \cup_{\left(D \nu_{\mathcal{I}}, 1\right)} D \Psi$. 

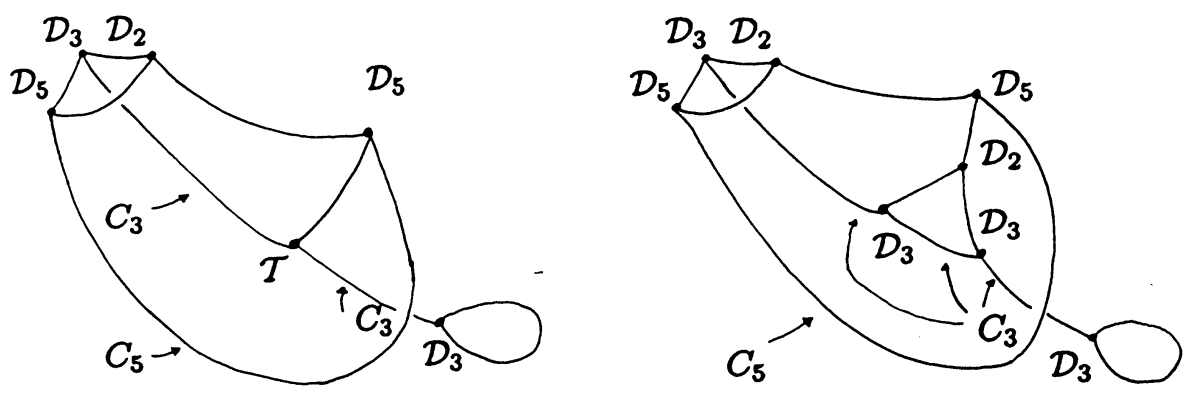

Singular set for $Q_{1}$.

Singular set for $Q_{2}$.

Figure 3.4.

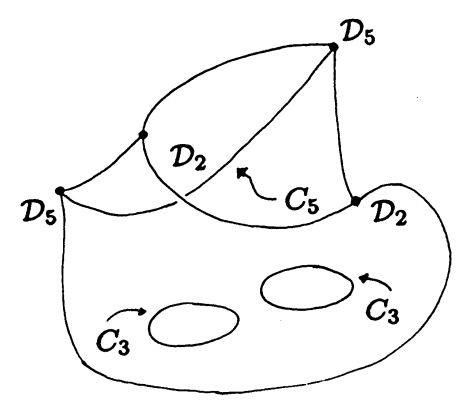

Singular set for $Q_{3}$.

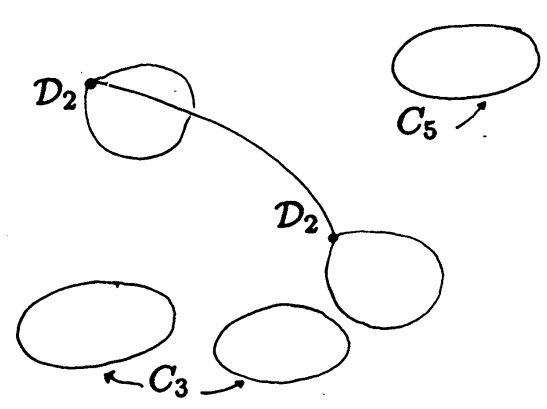

Singular set for $Q_{4}$.

Figure 3.5. 


\section{References}

[1] P.E. Conner and E.E. Floyd, Differentiable Periodic Maps, Springer, Berlin, 1964.

[2] K.S. Druschel, Oriented Orbifold Cobordism, Pacific J. Math., 164(2) (1994), 299-319.

[3] V.A. Rohlin, A three-dimensional manifold is the boundary of a four-dimensional one, Dokl. Akad. Nauk SSSR, 81 (1951), 355-357.

Received September 7, 1996 and revised June 29, 1998.

St. Louis University

ST. Louis, MO 63103

E-mail address: druschelks@slu.edu 